\title{
Study of gender differences in VR response following cardiac surgery.
}

\author{
Jose Luis Mosso ${ }^{1}$, Brenda K. Wiederhold ${ }^{2 *}$, Filippo La Paglia ${ }^{3}$, Danilo Guarino ${ }^{3}$, Daniele La Barbera ${ }^{3}$, Jose \\ Luis Mosso $\mathrm{Jr}^{4}{ }^{4}$, Ian Miller ${ }^{5}$, Mark D. Wiederhold ${ }^{6}$ \\ ${ }^{1}$ Clínica de Especialidades Alberto Pisanty Ovadía del ISSSTE, Hospital General y Regional \\ Numero 25 del IMSS, Mexico \\ ${ }^{2}$ Virtual Reality Medical Institute, Brussels, Belgium \\ ${ }^{3}$ Università degli studi di Palermo, Palermo, Italy. \\ ${ }^{4}$ School of Medicine, Universidad Anahuac, Mexico City, Mexico \\ ${ }^{5}$ Interactive Media Institute, San Diego, California \\ ${ }^{6}$ Virtual Reality Medical Center, La Jolla, California
}

\begin{abstract}
Cardiac and other invasive surgical procedures cause significant anxiety and stress to patients and their family members. In this study Virtual Reality (VR) was used as a method to reduce stress, anxiety and pain in patients undergoing surgical procedures. This study compared a cohort of patients in the interval prior to and successive to the surgery, differentiating the responses achieved by males and females and comparing the effectiveness. The results are encouraging: they demonstrate the efficacy of VR treatment and the safety of the method and detection of differences in the responses based on gender.
\end{abstract}

Keywords: Gender differences, Cardiac surgery, Anxiety, Stress, Surgery.

Accepted on March 20, 2017

\section{Introduction}

Despite the progress in anesthesia and surgical methods, a common problem in surgical procedures using local or regional anesthesia is that many patients experience high levels of anxiety both before and during the operation. Their anxiety often arises from a lack of information or knowledge about medical procedures and they presume that such procedures will cause them pain and discomfort. Local and regional anesthesia are techniques used to render part of the body insensitive to pain without affecting consciousness; this means that during the operation patients can perceive everything that is happening around them: they can see doctors and nurses moving around, hear their voices and comments and so on. Patients with high levels of anxiety usually perceive the procedure to be more distressing than it really is. Therefore, anxiety is a common problem for patients who undergo surgical procedures, often associated with a number of negative behaviors during and after the surgical experience.

Analgesics have been the mainstream solution for alleviating pain in the past. However, medications are often ineffective; some patients may experience high levels of pain after surgery, resulting in a high intake of extra analgesics, longer hospitalization, and slower recovery. Since drug treatments alone have frequently proven to be inadequate in reducig stress and anxiety in surgical contexts, there has been an increased interest in non-invasive supplementary medical therapies, including music $[1,2]$ relaxation, guided imagery, hypnosis, etc., to reduce pain and tension during pre and post operative phases. Different experimental studies have shown that distraction techniques are effective in reducing pain and related anxiety. Management of procedure-related distress commonly includes the use of distraction techniques that intend to divert attention away from the procedure and instead focus attention on an activity or task $[3,4]$. Prior research has shown Virtual Reality (VR) distraction to be beneficial for patients who are undergoing painful medical procedures [58]. It has also been shown that VR can be used in a sustained manner with positive results.

Virtual environments are safe alternatives to medications, as they eliminate risk of dependence on drugs and cause minimal side effects. These advances have shifted towards using distraction and hypnosis techniques to treat pain [9]. Demonstrated in its utility as a distraction technique, VR can be considered as an innovative form of e-health therapy gaining further recognition as a means of attenuating pain during medical procedures. VR reduces distress and pain perception by providing a form of immersive distraction that taxes the patient's limited attention capacity, resulting in the withdrawal of attention from the real, noxious, external stimulus with a subsequent reduction in pain and stress [10]. This technology allows the patient to explore and manage several situations inspired to daily experiences, using the real correspondent behaviors in a more controlled, safe and low-cost setting than in real life situations. In the last few years VR has been applied for the assessment and rehabilitation of several psychological diseases like Post-Traumatic Stress Disorder [11], anxiety [12], and eating disorders [13]. Furthermore, VR has been applied in neuropsychological domains like neglect 
Citation: Mosso JL, Wiederhold BK, Paglia FL, et al. Study of gender differences in VR response following cardiac surgery. J Psychol Cognition. 2017;2(1):67-71.

[14], executive functions [15] and cognitive rehabilitation of schizophrenia [16]. Therefore, these advanced systems interact at many levels with the VE, stimulating sights, sounds, and motion to encourage immersion in the virtual world to enhance distraction from pain [17].

Finally, different studies have demonstrated that VR can significantly reduce subjective pain intensity ratings and pain-related brain activity. Virtual reality has been shown to be effective in decreasing pain intensity and anxiety in the treatment of burns [18-20] and surgery [21-24], as well as in dental procedures [25]. In one controlled study, adult burn patients undergoing physical therapy reported less pain while immersed in VR than those who only participated in standard physical therapy [20]. Evidence shows that $\mathrm{VR}$ is effective in reducing pain in children with cancer. As chemotherapy-related symptom distress was reduced significantly immediately after using VR during treatment [26-28]. Another clinical study observed that dental patients undergoing plaque removal below their gum line experienced considerable reduction in pain when using VR compared to participants that watched a movie and to participants that did not have any type of distraction [29]. In a study conducted by Johnston in 1980, the natural course of anxiety before and after surgery was examined using the State Trait Anxiety Inventory in 4 studies involving 136 surgical patients. The results suggested that high levels of anxiety were experienced before admission to hospital, between admission and surgery and following surgery, and were not restricted to the immediate pre-operative period [30].

\section{Methods}

A group of 22 patients, composed of 7 women and 15 men underwent cardiac surgical interventions. They were given a VR treatment session before the surgery, and another VR session immediately following surgery. The VR scenarios used were "Enchanted Forest" and "Cliff", developed at The Virtual Reality Medical Center, La Jolla, California. The worlds help evoke relaxation and deep breathing [31]. To evaluate the patients' responses, both objective and subjective parameters were monitored. Objective measures included: heart rate, breathing rate, arterial blood gas analysis $\left(\mathrm{pH}\right.$, bicarbonates, $\mathrm{CO}_{2}, \mathrm{FiO}_{2}$, saturation of $\mathrm{O}_{2}$ ) and blood pressure values (systolic, diastolic, mean arterial pressure). A Likert scale was used to provide a subjective measure in reference to the pain experienced [32].

The Virtual Reality scenarios were presented through an eMagin Headmounted Display (HMD) that displayed 3D stereoscopic color images with a resolution of $1024 \times 768$ pixels. The auditory effects were delivered through binaural headphones. The computer was a Pentium IV, $3 \mathrm{GHz}, 2 \mathrm{~GB}$ Ram, NVIDIA QuadroFX 4500512 MB DDR3 Graphics card. Virtual scenarios were modeled and animated using 3D StudioMax, Adobe Photoshop and Maya. Navigation was conducted with a Logitech Joypad [31,32].

\section{Results}

Figure 1 describes differences between the B (before) and A

\begin{tabular}{|c|c|c|c|c|c|c|}
\hline & \multicolumn{3}{|c|}{ Female } & \multicolumn{3}{|c|}{ Male } \\
\hline & Before & After & Result & Before & After & Result \\
\hline $\mathrm{pH}$ & 7,415 & 7,413 & & 7,427 & 7,450 & \\
\hline Bicarbonate & 23,483 & 23,463 & & 28,587 & 29,160 & \\
\hline $\mathrm{CO} 2$ & 37,167 & 33,500 & & 35,200 & 35,333 & \\
\hline FiO2 & 87,833 & 79,500 & & 72,400 & 79,333 & \\
\hline BR & 19,5 & 14,5 & & 17,733 & 16,867 & \\
\hline HR & 87,71 & 85,29 & & 80,00 & 79,47 & \\
\hline Saturation & 95,86 & 95,71 & & 92,93 & 92,25 & \\
\hline Systolic & 117,57 & 121,29 & & 118,40 & 124,67 & \\
\hline Diastolic & 72,57 & 73,14 & & 72,20 & 71,80 & \\
\hline Mean Art Press & 87,57 & 89,19 & & 87,60 & 89,42 & \\
\hline Likert & 8,43 & 5,00 & & 7,80 & 3,00 & \\
\hline
\end{tabular}

Figure 1. Differences between the B (before) and A (after) values are shown, separated according to gender of the patients. 
(after) values, separated according to gender of the patients. The behavior is the opposite between the two sexes in 6 of the 11 cases. In particular it is the opposite in all four detections regarding Arterial Blood Gas analysis.

\section{Focus on ABG analysis}

In women, we see a marked reduction of $\mathrm{CO}_{2}$ and $\mathrm{FiO}_{2}$, while bicarbonate and $\mathrm{pH}$ values show marginal changes. Breathing Rate (BR), a factor that can drastically influence the acid/base equilibrium, shows a significant reduction of the respiratory rate. This could cause an acidification of the blood, due to retention of $\mathrm{CO}_{2}$, but this seems not to be the case. In females, even with a reduction of $25 \%$ of their respiratory rate, there is no increase of the $\mathrm{CO}_{2}$ values; rather we identified a significant reduction. Furthermore, nothing suggests a change in metabolic parameters regarding the acid/base equilibrium, since the bicarbonate is stationary in physiological values.

Male patients behave differently in that they exhibit an increase in their $\mathrm{pH}$ value of 0,023 . The differences between $\mathrm{B}$ and $\mathrm{A}$ are not high in these patients, except for the $\mathrm{FiO}_{2}$ value which has a significant increase over time. Breathing rate decreased by 0.866 while $\mathrm{CO}_{2}$ increased by 0.133 and bicarbonate value increased by 0.573 , suggesting that in male patients there are no significant changes in the main factors of acid/base equilibrium (Figure 2).

\section{Other objective measures}

Heart rate, oxygen saturation and blood pressure show no difference between genders. The behavior is almost equal indeed, with the exception of diastolic pressure, which increases in females and decreases in males. The heart rate decreases more in females $(-2,51 \mathrm{acts} / \mathrm{min})$ than in males $(-0,53 \mathrm{acts} / \mathrm{min})$, indicating relaxation in both genders after the procedure.

The saturation remains almost unvaried in both sexes (a very little decrease can be noticed), staying in optimal physiological range in women, and a little below the normal range in men. The pressure values behave in a similar way, with the exception of the diastolic pressure:

- Systolic pressure increases in both sexes, but more in men. Women experience an increase of $3,75 \mathrm{~mm} \mathrm{Hg}$, while men have a more substantial increase, which is 6,27 mm Hg;

- Diastolic pressure increases in women while it decreases in men;

- Mean arterial pressure increases in both sexes in almost

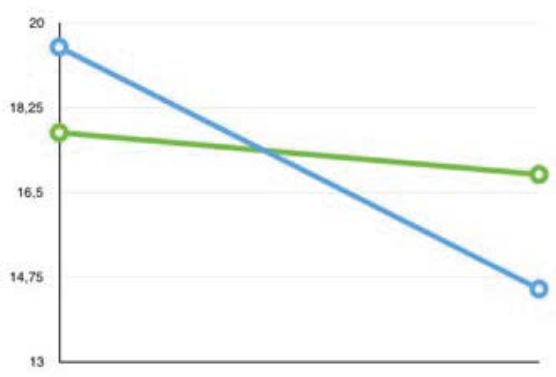

Breathing rate

Figure 2. Breathing rate.

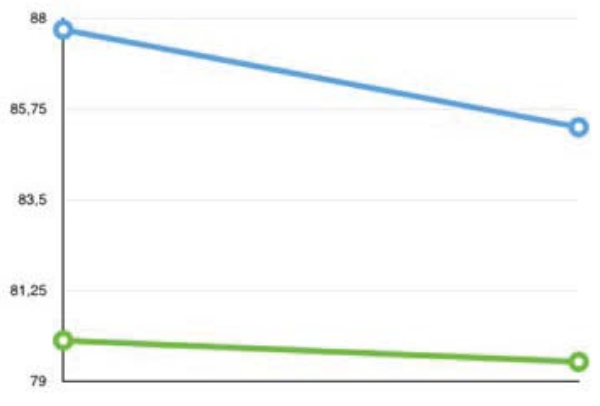

F: -5 acts $/ \mathrm{min}$

M: $-0,9$ acts/min

Figure 3. Heart rate.

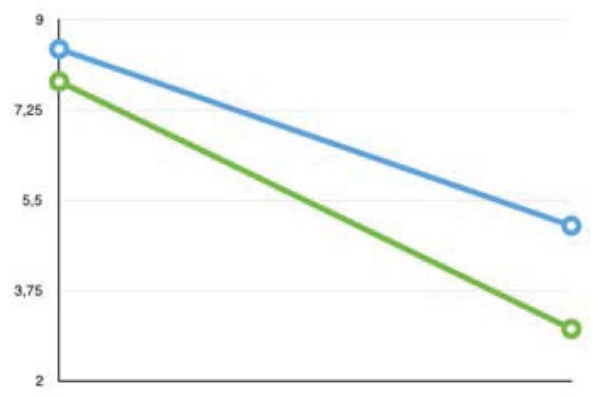

Likert scale

$F:-3,43$ score

M: $-4,8$ score

Figure 4. Likert scale. 
the same amount: 1,62 for women and 1,82 for men.

Males and females had similar mean arterial pressure values; however, men had higher systolic pressure when compared to women. We noted that diastolic pressure decreases in men while it remains stable in women (Figure 3).

\section{Likert scale (pain)}

There's a clear and impressive decrease of the Likert values in both sexes. We have a $-3,43$ in women and $-4,80$ in men, suggesting that there is a significant decrease in pain perception in both male and female subjects (Figure 4).

\section{Discussion}

Very few studies have examined gender differences in physiological responses to VR. In this study we show that $\mathrm{VR}$ is an effective medium to reduce stress and anxiety in patients undergoing cardiac surgery. Some studies on gender differences in pain threshold have produced interesting results. For example, an investigation of gender differences in experimental pain threshold detected significantly lower tolerance in females than males [33]. In this study, 240 participants (120 males and 120 females) received gradually increasing amounts of pressure in the dorsal interosseous muscle. In comparing time of tolerance and the average amount of pressure applied to each subject, the experimenters concluded that females exhibit lower thresholds.

Interesting differences in physiological response will be further investigated in future studies. We are as of yet unable to correlate gender differences in physiological response to clinical outcome. Our study suggests we further study these differences, as we continue to promote individualized therapy for all patients. Additional studies support the notion of gender differences in perceived pain. Bingefors and Isacson found major differences in reported pain between 5,000 males and females in Uppland, Sweden. Surveying the population about pain problems that effect their work, daily living, and social life, the researchers concluded that the prevalence of pain conditions was higher among women than men [34]. Comparisons of genders in thermal sensitivity also report variations between males and females in thermal sensation response [35]. These examples suggest gender differences in pain and highlight an increasing need for effective, accessible, and easy to use devices that control pain perception.

\section{Conclusion}

This brief study confirms the efficacy of VR in helping patients reduce stress before, during and after medical and surgical procedures. We anticipate additional positive effects in reducing these often harmful symptoms that can affect both quality of life and activities of daily living. We are interested in continued investigation and are working toward making this intervention more effective, less expensive and available across platforms to include mobile healthcare and behavioral health.

\section{References}

1. Nilsson U. The anxiety and pain reducing effects of music interventions: A systematic review. AORN J. 2008;87(4):780-807.
2. Lee $\mathrm{KC}$, Chao $\mathrm{YH}$, Yiin JJ, et al. Effectiveness of different music-playing devices for reducing preoperative anxiety: A clinical control study. Int J Nurs Stud. 2011;48(10):1180-7.

3. Hudson BF, Ogden J, Whiteley MS. Randomized controlled trial to compare the effect of simple distraction interventions on pain and anxiety experienced during conscious surgery. Eur J Pain. 2015;19(10):1447-55.

4. Umezawa S, Higurashi T, Uchiyama S, et al. Visual distraction alone for the improvement of colonoscopyrelated pain and satisfaction. World J Gastroenterol. 2015;21(15):4707.

5. Mosso, JL, Wiederhold BK, Miller I, Dejanir L, Wiederhold MD. Virtual reality assisted anesthesia (VRAA) during upper gastrointestinal endoscopy: Report of 115 cases - Analysis of physiological responses. Surgical Research Updates. 2017;5.

6. Mahrer NM, Gold JI. The use of virtual reality for pain control: A review. Curr Pain Headache Rep. 2009;13:1009.

7. Malloi KM, Milling LS. The effectiveness of virtual reality distraction for pain reduction: A systematic review. Clin Psychol Rev. 2010;8:1011-8.

8. Wiederhold MD, Wiederhold BK. Virtual reality and interactive simulation for pain distraction. Pain Med. 2007;8:S182-8.

9. Schmitt YS, Hoffman HG, Sharar SR. A randomized, controlled trial of immersive virtual reality analgesia during physical therapy for pediatric burn injuries. Burns. 2011;37:61-8.

10. Vázquez JL, Santander A, Gao K, et al. Using cybertherapy to reduce postoperative anxiety in cardiac recovery intensive care units. J Anesth Clin Res. 2013;4:363.

11. McLay RN, Wood DP, Webb-Murphy JA, et al. A randomized, controlled trial of virtual reality-graded exposure therapy for post-traumatic stress disorder in active duty service members with combat-related posttraumatic stress disorder. Cyberpsychol Behav Soc Netw. 2011;14(4):223-9.

12. Wiederhold BK, Wiederhold MD. Virtual reality therapy for anxiety disorders: Advances in education and treatment. American Psychological Association Press, New York. 2004.

13. Wiederhold BK, Riva G, Gutierrez-Maldonado J. Virtual reality in the assessment and treatment of weight-related disorders. Cyberpsychol Behav Soc Netw. 2016;19(2):6773.

14. Pedroli E, Serino S, Cipresso P, et al. Assessment and rehabilitation of neglect using virtual reality: A systematic review. Front Behav Neurosci. 2015;9-226.

15. La Paglia F, La Cascia C, Rizzo R, et al. Cognitive assessment of OCD patients: NeuroVR vs. 
neuropsychological test. Stud Health Technol Inform. 2014;199:40-4.

16. La Paglia F, La Cascia C, Rizzo R, et al. Cognitive rehabilitation of schizophrenia through NeuroVR training. Stud Health Technol Inform. 2013;191:158-62.

17. Mosso-Vazquez JL, Gao K, Wiederhold BK, et al. Virtual reality for pain management in cardiac surgery. Cyberpsychol Behav Soc Netw. 2014;17(6):371-8.

18. Das DA, Grimmer KA, Sparnon AL, et al. The efficacy of playing a virtual reality game in modulating pain for children with acute burn injuries: A randomized controlled trial. BMC Pediatr. 2005;5:1.

19. Hoffman HG, Patterson DR, Seibel E, et al. Virtual reality pain control during burn wound debridement in the hydrotank. Clin J Pain. 2008;24(4):299-330.

20. Hoffman HG, Patterson DR, Carrougher GJ. Use of virtual reality for adjunctive treatment of adult burn pain during physical therapy: a controlled study. Clin J Pain. 2000; 16: 244-250.

21. Vazquez JLM, Santander A, Gao K, Wiederhold $\mathrm{B}$, Wiederhold MD. Using cybertherapy to reduce postoperative anxiety in cardiac recovery intensive care units. J Anesth Clin Res. 2013; 4: 363.

22. Mosso JL, Rizzo S, Wiederhold B, et al. Cybertherapy new applications for discomfort reductions. Surgical care unit of heart, neonatology care unit, transplant kidney care unit, delivery room-cesarean surgery and ambulatory surgery, 27 case reports. Stud Health Technol Inform, 2007; 125: 334-336.

23. Mosso-Vasquez JL, Wiederhold BK, Miller I, Wiederhold MD. Virtual reality assisted Anesthesia (VRAA) during upper gastrointestinal endoscopy: Report of 115 cases. EMJ Innov. 2017; 1(1): 75-82.

24. Mosso JL, Rizzo S, Wiederhold B, Lara V, et al. Cybertherapy new applications for discomfort reductions. Surgical care unit of heart, neonatology care unit, transplant kidney care unit, delivery room-cesarean surgery and ambulatory surgery, 27 case reports. Stud Health Technol Inform. 2006; 125: 334-6.

25. Wiederhold MD, Gao K, Wiederhold BK. Clinical use of virtual reality distraction system to reduce anxiety and pain in dental procedures. Cyberpsychol Behav Soc Netw. 2014; 17(6): 359-65.

26. Schneider SM, Prince-Paul M, Allen MJ, Silverman $\mathrm{P}$, Talaba D. Virtual reality as a distraction intervention for women receiving chemotherapy. Oncol Nurs Forum. $2004 ; 3: 81-88$.

27. Schneider S, Kisby C, Flint E. Effect of virtual reality on time perception in patients receiving chemotherapy. Supportive care cancer. 2011; 19: 555-64.

28. Schneider SM, Wokrman ML. Virtual reality as a distraction intervention for older children receiving chemotherapy. J Pediatr Nurs. 2000;26:593-7.

29. Hoffman HG, Garcia-Palacios A, Patterson DR, et al. The effectiveness of virtual reality for dental pain control: A case study. Cyberpsychol Behav. 2001;4:527-35.

30. Johnston M. 'Anxiety in surgical patients'. Psychol Med. 1980;10(1):145-52.

31. Wiederhold B. Virtual reality as a distraction technique in chronic pain patients. Cyberpsychol Behav Soc Netw. 2014;17(6):346-52.

32. Uebersax JS. 'Likert scales: Dispelling the confusion'. John Uebersax Enterprises LLC. 2006.

33. Chesterton LS, Barlas P, Foster NE, et al. Gender differences in pressure pain threshold in healthy humans. Pain. 2003;101(3):259-66.

34. Bingefors K, Isacson D. Epidemiology, co-morbidity and impact on health-related quality of life of selfreported headache and musculoskeletal pain-a gender perspective. Eur J Pain. 2004;8(5):435-50.

35. Gerrett N, Ouzzahra Y, Coleby S, et al. Thermal sensitivity to warmth during rest and exercise: A sex comparison. Eur J Appl Physiol. 2014;114(7):1451-62.

\section{*Correspondence to:}

Brenda Wiederhold

President

Virtual Reality Medical Center

6540 Lusk Boulevard, Suite C115

San Diego, California 92121

Tel; 18586420267

E-mail: b@vrphobia.eu 